ISSN: 0213-3563

http://dx.doi.org/10.14201/azafea201921159179

\title{
SOBRE LOS MODELOS ENCUBIERTOS DE ANTROPOCENTRISMO EN NUESTRA RELACIÓN CON EL MEDIO ORGÁNICO ${ }^{1}$
}

\author{
On the undercover models of anthropocentrism in our relationship \\ with the organic environment
}

\section{Guillermo Aguirre Martínez \\ Universidad de Deusto}

Recibido: 13 de junio de 2018

Aceptado: 26 de marzo de 2019

\section{RESUMEN}

Estas páginas pretenden abordar conceptos relativos a la bioética a partir de la revisión de nuestra concepción de los conceptos de sensibilidad e inteligencia en el reino vegetal, cuestiones ambas que no dejan de comprenderse como umbral en el que queda a un lado un deseo de respeto hacia el medio con el que convivimos y, en el otro, de modo más sutil, un nuevo apropiamiento de carácter antropocéntrico por parte del sujeto.

Palabras clave: bioética, Inteligencia, sensibilidad, medio ambiente.

\begin{abstract}
Throughout the next pages we will study some aspects in relation with the concept of sensitivity and intelligence in the plant kingdom and in the human being. These aspects remain at a point in the middle of a fork: the respect towards the environment in the one side and, in the other one, a way of anthropocentric knowledge.
\end{abstract}

Keywords: Bioethical, Intelligence, Sensitivity, Environment.

1. La realización de este artículo ha sido financiada con un Contrato Juan de la Cierva - Incorporación, concedido por el Ministerio de Economía, Industria y Competitividad (IJCI-2015-26934). 
GUILLERMO AGUIRRE MARTÍNEZ

SOBRE LOS MODELOS ENCUBIERTOS DE ANTROPOCENTRISMO EN NUESTRA RELACIÓN CON EL MEDIO ORGÁNICO

\section{INTRODUCCIÓN}

El eje espacio-temporal determina nuestra percepción y nuestro estar en y ante la realidad: sobre esto no hay nada que añadir dado que sin aquellos dos no hay realidad al menos en un sentido acostumbrado. Cuanto, en cambio, se pretende exponer con este artículo, remite a consideraciones en torno a nuestra comprensión de la vida, de lo vivo, con base a dichos a priori kantianos y más allá de que cada uno considere que lo real reside en el sujeto, en el objeto percibido por el sujeto, o en un tercer factor del que ambos toman parte o coincidente con ellos.

El fenómeno que deseamos observar se apoya en la proliferación de lecturas remitentes a un modo de sensibilidad e inteligencia en el reino vegetal. ${ }^{2} \mathrm{La}$ adjudicación de ambas cualidades, sensibilidad e inteligencia, a los organismos

2. Citamos, entre otros, a Mancuso por su actualidad, a Francis Hallé -desde lo expuesto en su Elogio de la planta- por su envergadura, o a Paco Calvo por tratarse de una referencia en nuestro país en el ámbito de la neurobiología.

Como contrapunto a estas ideas, encontramos, una más entre tantas voces, las siguiente palabras de Rob DeSalle e Ian Tattersall: "Los biólogos europeos František Baluška y Stefano Mancuso abogaban enérgicamente por la existencia en sentido literal de un sistema nervioso en las plantas, y proponían que 'la eliminación del viejo cisma aristotélico entre plantas y animales unificará todos los organismos pluricelulares bajo un mismo paraguas conceptual'” (Desalle, R. / TATTERsall, I., El cerebro. Big Bangs, comportamientos y creencias, Barcelona, Galaxia Gutenberg, 2017, p. 95). La crítica aquí la encontramos tanto en el aludido 'sentido literal', como en la mezcolanza que derivaría de ese único paraguas conceptual.

La lasitud que los aludidos autores reprochan a Mancuso la apreciamos en argumentos como el que sigue, tan reales como, entre comillas, acientíficos a un tiempo: "Todos los olores producidos por los vegetales [...] equivalen a un mensaje concreto: son las 'palabras' de las plantas, su vocabulario" (Mancuso, S., Sensibilidad e inteligencia en el mundo vegetal, Barcelona, Galaxia Gutenberg, 2015, p. 48).

La cuestión de la existencia de un sistema nervioso en las plantas, por lo que conozco, fue ya tratada desde parámetros científicos por Darwin y otros biólogos de la época. Poco después, una naturaleza omniabarcante y acientífica stricto sensu como Strindberg, a la par que realizaba sus estudios sobre química, alquimia y óptica, entre otros, se presentará como firme impulsor del desarrollo de la idea de un sistema nervioso en el reino vegetal. Alguna de sus consideraciones al respecto las expone en su breve texto: ¿Dónde están los nervios en las plantas?, de 1896. Dentro del citado texto leemos rotundos ataques contra la líneas atrás mencionada distinción Aristotélica: “Todo esto está tan mal investigado y es tan poco conocido que para estudiar actualmente la botánica uno está obligado a leer libros de agricultores, jardineros y farmacéuticos para obtener alguna idea sobre cómo trabaja la naturaleza, lo cual es mucho más informativo que los auxiliares de laboratorio en los departamentos de biología" (STRINDBERG, A., Una mirada al Universo, Madrid, Siruela, 2016, p. 101). 
de este reino, nos pone a su vez en relación con aspectos afines a nuestra concepción de términos como 'respeto' o 'dignidad', conceptos más y más pertinentes de ser revisados a medida que, en lo tocante al tema que nos ocupa, establecemos nuevas identificaciones entre realidades hasta ahora relativas al sujeto y el objeto de estudio -el reino vegetal-, traspasándose con ello la frontera de lo humano a la hora de aplicar conceptos como los aludidos. De ello hablaremos más adelante. Estos términos - respeto y dignidad-, por lo común y dentro del marco de la civilización occidental, habitualmente han sido reservados al ser humano, a sus dioses, así como a un grupo selecto de animales, precisamente aquéllos más afines, por unos u otros motivos, a nuestra condición natural. En este punto, comprendemos, allí donde no hay identificación con el objeto, se nubla, no nos es posible hallar, un algo a preservar de la aludida dignidad del organismo observado.

Los factores, por tanto, que nos permiten aplicar e identificar nuestros códigos éticos a la realidad adjudicada a otros organismos, pasan por un giro antropocéntrico desde el que valoramos realidades no humanas a partir de cualidades humanas. Esto supone, en definitiva, que el respeto mostrado en este caso hacia uno u otro organismo del reino vegetal -del mismo modo que más comúnmente se ha dado a lo largo de la historia hacia parte del mundo animal- viene de la mano de una empatía cuyo origen -generalizamos- radica en su afinidad con el individuo. A la hora de realizar esta identificación se produce un movimiento de aproximación desde ambos frentes si bien, en este caso, determinado exclusivamente por el sujeto que ejerce tal ponderación. El objeto de estas apreciaciones, en el caso con el que ejemplificamos

Destaco, además, dentro del ámbito afín a la Antroposofía, las ideas de Frits H. Julius expuestas en su trabajo Metamorfosis -alabado por Hallé, diremos por si con ello se apuntala algo su enfoque-. En la línea abierta por Goethe y Schiller -por Goethe en mayor medida y desde luego de modo más empírico- en sus estudios sobre la forma como expresión de una voluntad primordial, y posteriormente por Steiner, Julius enfoca los conceptos de sensibilidad e inteligencia en el mundo vegetal a partir de la idea -tan propia del ideario de Schiller- de voluntad. En este sentido, a partir del concepto de forma y formación compartido con Hallé, Haeckel y D’Arcy Thompson, resultan reveladoras las relaciones que Julius establece entre aquellas dos -forma y formación- y los conceptos de inteligencia y sensación: "Lo que en la planta es tendencia vertical, se proyecta en el hombre como el apremio de determinar las cosas por la propia voluntad, o de apropiárselas por el propio pensamiento. La tendencia periférica, a su vez, se convierte en el hombre en propensión a entregarse al mundo ambiente por la sensación y el sentimiento” (Julius, F. H., Metamorfosis. Claves para la comprensión del desarrollo vegetal y de la vida bumana, Buenos Aires, Antropomórfica, 2008, p. 98). 
remitente a los organismos del reino vegetal, requerirá de la atribución de cualidades humanas próximas en este caso a nuestro concepto de inteligencia y sensibilidad. ${ }^{3}$ El individuo, en perfecta simetría, situará la esencia o la característica identificadora de lo humano no en un orden racional sino en uno sensorial o, en todo caso, en un estrato más próximo a facultades vegetativas que a aquellas otras intelectivas, lo que implicará, a fin de cuentas y en los que a nosotros nos interesa, designar la cualidad intelectiva con base a factores cada vez más alejados de una lógica racional. Este proceder, cabe advertir, esta búsqueda de semejanzas, resulta natural en el individuo no sólo en lo que concierne a otros reinos sino asimismo en lo tocante a su relación con el resto de sujetos. Con ello, por experiencia histórica, advertimos a su vez que ahí donde se da una relación de empatía se esconde un deseo profundo de asimilación o de apropiación del objeto observado.

El grado de respeto, de dignidad, por tanto, que mostramos en este caso hacia organismos del reino vegetal, pasa por la demostración de sensibilidades parejas a la nuestra o, en cualquier caso, digeribles por nuestro razonamiento, dándose en ello tanto una reconsideración de conceptos como sensibilidad e inteligencia en su aplicación tanto al sujeto de estudio como al objeto observador. La aludida equiparación, claro está, no identificará funcionamientos racionales sino respuestas comprendidas como intelectivas en función de capacidades hasta entonces dejadas de lado ya sea por considerarse secundarias, ya por desconocerse la naturaleza de éstas o incluso su existencia. Por todo ello, aun disponiendo el sujeto de una capacidad lógicoracional no hallable en el orden vegetal al menos en los términos que nos son comunes, se podrá llegar a considerar dicha capacidad, desde esta nueva

3. Señala Mancuso al respecto: "Podemos imaginar fácilmente una inteligencia que no sea fruto de la actividad de un único órgano; la inteligencia es connatural a la vida, haya o no haya cerebro. Las plantas, desde este punto de vista, son la prueba más evidente de que el encéfalo es un 'accidente' que se ha desarrollado tan sólo en un reducido número de seres vivos, los animales, mientras que en la inmensa mayoría de organismos -los vegetales- la inteligencia se ha desarrollado sin que exista ningún órgano específico” (Mancuso, S., El futuro es vegetal, Barcelona, Galaxia Gutenberg, 2017, p. 21). En última instancia, Mancuso, en relación con un modo de inteligencia vegetal, apunta que "se trata de una cuestión terminológica y depende de la definición de inteligencia que elijamos" (Op. Cit., 2015, p. 7), pues "si definimos la inteligencia como la capacidad para dar respuesta a problemas, entonces no es en modo alguno posible trazar una divisoria artificial por encima de la cual despunta la inteligencia y por debajo de la cual sólo hay autómatas (es decir, seres que responden a los estímulos ambientales de manera automática)" (Ibid., p. 112). 
perspectiva propuesta desde un sector de la neurobiología, como herramienta propia del ser humano diferente pero no superior a la de aquellas otras empleadas en el reino vegetal comprendidas como sensitivo-intelectivas. Cada reino, pues, participa de unas óptimas capacidades análogas en sus resultados y funciones -no en el modo en que se ejecutan o en su funcionamiento propiamente dicho-. Así visto -considerará este sector de la neurobiología que no deja de ser un vértice más del nuevo paradigma en el que más adelante nos detendremos-, cuanto aquí acontece apunta a una mera cuestión de diferencia, de adaptación si se quiere, no de cualidad o capacidad, de manera que funciones apenas desarrolladas, ineficientes o directamente inexistentes en la realidad del ser humano, se dan en cambio en las plantas en grado efectivo, $y$ viceversa. Situar unas por encima de otras, se añadirá aún, supone olvidarse de la situación y validez de cada término en la naturaleza, supone romper desde una perspectiva antropocéntrica la relación de conjunto. ${ }^{4}$ Más adelante aclararemos nuestra consideración al respecto.

Se trata, como vemos, de un fenómeno de apasionante interés con vistas a definir nuestra idea de individuos, la concepción de nuestro mundo circundante, así como nuestro futuro como especie a medio plazo -entroncando de lleno esta deriva con el concepto de posthumanismo: si bien entrar en este terreno nos llevaría, en el marco de este artículo, a desplazarnos demasiado lejos- ${ }^{5}$ Ahí donde nos encamina este entendimiento, a fin de cuentas, es hacia una visión del sujeto menos individualizada, más común y participativa con el medio. No sólo la especie sino lo orgánico en general se impone sobre el sujeto si bien habría que comprender en primer lugar en qué nos reconocemos como individuos o, tomando de nuevo como apoyo una cosmovisión

4. No comprendemos necesario detenernos en las variantes encontradas en los biólogos consultados -siendo Mancuso el máximo defensor de una asimilación entre inteligencia y sensibilidad en el reino vegetal y el animal- dado que aquí cuanto interesa es la cuestión de fondo, esto es, cómo afecta esta comprensión a nuestra relación con el medio -vegetal en primer lugar-, y qué grado de falseamiento puede haber en este deseo de asimilación.

5. Una visión panorámica de la cuestión acompañada de una serie de interrogantes a modo de puntos o pautas de reflexión, se recoge en el reciente trabajo de Antonio Diéguez: Transhumanismo, en el que alude -y esto se presenta interesante con vistas al cuestionamiento que aquí realizaremos en torno a los conceptos de vida, muerte e individuo-, a una "resurrección computacional de los muertos" (DiÉGuez, A., Transhumanismo, Barcelona, Herder, 2018, p. 56), justo después de citar las siguientes palabras proféticas -en un texto fechado en 1986- de Hans Moravec: "Será nuestra progenie intelectual, no genética, la que heredará el universo". 


\section{GUILLERMO AGUIRRE MARTÍNEZ
164 SOBRE LOS MODELOS ENCUBIERTOS DE ANTROPOCENTRISMO EN NUESTRA RELACIÓN CON EL MEDIO ORGÁNICO}

posthumanista o posthumana, de qué especie hablamos o hablaremos en venideros años.

\section{Colonización moralmente Permisible}

A la hora de derivar el concepto de dignidad hacia un ámbito vegetal aun, evidentemente, siempre a partir de una medida humana - de esta esfera no podemos salir, del mismo modo que no podríamos comprender que nosotros mismos podemos carecer de dignidad a ojos de un tercero racional o provisto, a su manera, de facultades intelectivas por mucho que no casen con nuestro concepto de racionalidad-, hemos de rendirnos ante la realidad de nuestra imposibilidad de asimilar categorías elementales a nuestra comprensión tales como vida, muerte o individuo, a condiciones existenciales distintas a la nuestra, dado que aquello que percibimos desde nuestros parámetros conceptuales nos define a nosotros y no ya a aquellos organismos en cuya existencia tales términos no poseen la realidad que nosotros les adjudicamos en nuestro afán por proyectar nuestra unidireccional perspectiva. Siendo estos términos útiles para explicar nuestra relación con el medio, escapan en un rizo paradójico a nuestro entendimiento en sí a la hora de aplicarlos a otras naturalezas.

Este hecho, para bien y para mal, desequilibra, desestructura, nuestras concepciones habituales establecidas en torno a nuestro mundo circundante, especialmente en lo que concierne al ámbito cultural de Occidente -esto es, Occidente y allí donde se ha impuesto su cosmovisión-. En este punto, podemos aclarar, se abre una brecha a la hora de interpretar la realidad por parte de una cultura altamente tecnificada y sometida al dominio abusivo de la razón, y cuanto acontece en este mismo caso por parte de otra cultura más asentada sobre modelos no orientados, en todas sus proyecciones, hacia lo cuantificable y sí hacia el equilibrio entre el sujeto y su entorno. No nos resulta aún inusual oír hablar de estas culturas desde términos relativos a lo primitivo o lo acultural. Y sin embargo, en lo que toca a nuestro tema, basta con acudir a un modelo de religiosidad propio de estas últimas sociedades no estructurado por una lógica sino por relaciones de empatía, no ya por una organización categórica rígida sino más bien por un rechazo de éstas, para advertir que allí donde se desarrolla un panteísmo o un pan-naturalismo capaz de comprender cada sustancia real o espectral, cada poso de existencia e inexistencia, como respetable y poseedora de dignidad, se da ya, sin necesi- 
dad de acogerse a las capacidades del objeto observado o presentido, sin necesidad por tanto de acudir a una interpretación empírico-racional en primer lugar, un respeto inherente a ese distinto modo de participación en el mundo: una visión y participación no tan arraigada a la propia concepción del yo, a nuestro ensimismamiento particular, y sí, en cambio, a una comprensión de la relación con el objeto observado enteramente desde sí. Cuanto se da es una sustitución, un deponer nuestra necesidad tanto de explicar la posición del medio en relación con nosotros, como incluso de defenderlo, y priorizar en cambio un estar en el medio, en relación con el entorno, sin salirnos de nuestros límites: es ésta, a fin de cuentas, la hybris desde la que Occidente se desfonda.

Por consiguiente y según lo expuesto, ahí donde el individuo de occidente necesita de una prueba científica para no colonizar a otras especies o para colonizarlas sutilmente y sin emborronar su conciencia, una cultura no asentada sobre un paradigma cientificista tenderá naturalmente a verse como parte del medio y no como individuo situado frente a aquél, no como sujeto poseedor de poder sobre aquél -tanto para explotarlo como para preservarlo- sino como actuante junto a aquél dentro de unos límites no morales sino naturales -separando un tanto forzadamente ambos conceptos-. En este sentido, por lo tanto, se comprende más necesaria la tarea de no hipertrofiar nuestra posición de poder, nuestra capacidad para absolver, condenar, permitir, decidir o conceptualizar, que la de nivelarnos desde nuestra base orgánica para en este terreno permanecer en adelante. No se trata ya, por tanto, de negar las capacidades de dominio del individuo, sino de no someterse a ellas como si en ellas se encontrase el núcleo de nuestra identidad. ${ }^{6}$ Cuanto se quiere reivindicar es un comportamiento, por tanto, no maniqueo, esto es, un no llegar al punto de comprendernos como seres no actuantes con el sistema a la hora, por ejemplo, de proveernos de alimento animal o vegetal. Se parte, en fin, de una consideración del sujeto como ser dotado de unas capacidades más o menos favorables respecto del resto de especies con vistas a la consecución de unas necesidades. Dicho de otro modo, querer convertirnos en jueces naturales, querer alejar la muerte de nuestra relación con el medio -recordemos que si bien no es éste, al menos no necesariamente, el afán de la nueva

6. Esto no implica el que, evidentemente, nuestra existencia dependa enteramente de un cierto equilibrio con el medio e incluso que, como reitera Mancuso, seamos organismos especialmente dependientes en la cadena orgánica. A mayores necesidades, y el individuo, al margen de las propiamente naturales, no deja de crearse más y más necesidades de carácter cultural, más dependiente es el organismo en cuestión. 
biología, sí determina cada día más y más la mentalidad de un cierto sector de la población-, se presenta como una nueva proyección de nuestro propio afán de inmortalidad, una nueva ramificación -terrible dada la neurosis que esconde y que domina este objetivo- de nuestra hipertrofiada hybris.

Este maniqueísmo o puritanismo a gran escala, no podemos dejarlo de lado, esconde un hondo sentimiento de culpa en la conciencia occidental. A mayor poder, mayor culpabilidad y, en consecuencia, mayores restricciones morales, mayor neurosis por tanto en cuanto que se cercena precisamente la parte natural y no la racional. Este corte entre lo natural y lo racional se comprende como necesario a la hora de exponer nuestras ideas aun cuando sin duda resulta forzado. Una hipertrofia científico-técnica contraatacada mediante restricciones morales sólo acentuará la potencia de aquélla, siendo este freno el que, aun aludiéndose a un punto de partida orgánico, actúa con singular fuerza en la asimilación por parte de la población de los preceptos de la nueva biología. La posibilidad de frenar dicha hipertrofia, de ser una opción real, no reside en una mayor presión por parte del término opuesto, esto es, no ha de partir de una guerra de uno contra sí mismo, contra la propia naturaleza -pues, ahora sí, en tanto que el razonamiento y sus derivados constituyen en verdad una fuerza natural, resulta irrefrenable desde el exterior-, sino en atender nuestras necesidades desde una limitación determinada por nuestra naturaleza orgánica o vegetativa -más dada a apagar su sed con unos mínimos elementales- y no desde aquella otra racional -más voraz y amenazante-: el par actuante se equilibra en cierto modo. Un factor determinante, en cualquier caso, queda como gran problema en este conflicto, y no es otro que la superpoblación: para ello sólo encontramos una solución desde los avances, justamente, técnicos. Tocamos sin embargo otro tema de estudio de modo que regresamos al nuestro. No hallaremos sin embargo tal fanatismo, en relación con la actitud expuesta, en sociedades no tecnificadas -salvo en el caso de quedar éstas sujetas a órdenes religiosos en los que rija la dialéctica culpa/castigo-, no encontraremos culto, por ejemplo, a un régimen vegano en la sabana ni en ningún espacio donde no reine un moralismo hipócrita desde su base pues, o bien desnaturaliza al sujeto, o bien hipertrofia su ambición y sed de apropiación. En estos tipos de pautas cuanto en primer lugar se advierte, con todo, apunta a la reparación de un sentimiento de culpa.

Tras este exordio, axial por otro lado en nuestra comprensión del problema, cabe decir que el respeto que en unas situaciones se produce como consecuencia o en compañía de un hallazgo científico -en este caso arraigado a la asimilación de facultades sensitivas e intelectuales en algún punto similares 
a las nuestras- y cuya valoración final permanece en el alambre hasta que se ratifica el nuevo punto de vista de modo legal o en todo caso por convención social, en otras situaciones obedece a un vínculo natural. Se da así una diferencia notoria: en un caso se respeta el medio por un motivo racional y moral, por una actitud en apariencia sensata que, en su hipertrofia, revela una represión en relación con la culpa: el sujeto participa de una condición natural que rechaza aun cuando ello supone un enfrentamiento entre dicha condición y sus determinaciones racionales. Su respuesta, por tanto, le conduce a rechazar su condición natural, animal, hiperhumanizándose si así queremos comprenderlo. En lo que respecta al segundo caso mencionado la relación con el medio se presenta más permisible y se da una aceptación y respeto por igual de la vida y de la muerte, de cierto poder y cierta sumisión, sin que ello rompa el equilibrio de la cadena.

Así por tanto, y hablamos ya de nuestra sociedad, observamos cómo incluso en nuestro modo de respetar cuanto en primer lugar realizamos concierne a un giro antropocéntrico desde el que calibramos la realidad del objeto. Esto para nuestro asombro se da incluso en aquellos momentos en que, con la mejor intención del mundo, se busca participar de una mirada no antropocéntrica capaz de acercarnos a la realidad con objeto de dotarla de valor en sí y no para nosotros. Más sorprendente nos resultará, en lo concerniente a sociedades escasamente tecnificadas, que su comprensión integradora de la existencia no derive o no camine necesariamente de la mano de un respeto por la vida del objeto observado al menos de acuerdo con nuestra comprensión de los conceptos de 'vida' y 'respeto'. Nada de esto encajará en nuestras estructuras de pensamiento y costumbres en tanto que en estas otras culturas, precisamente desde su particular entendimiento de dignidad o sacralidad, tal respeto puede llamar a deponer la vida del organismo dignificado ya sea por mera necesidad o, lo que a nosotros nos ha de parecer más terrible, por comprenderse, precisamente, su existencia como sagrada, ya se trate de un animal, un dios, un tótem, una planta o un individuo.

En estos casos asistiremos perplejos a una comprensión de concepciones tales como la vida y la muerte, el respeto, la dignidad y la sacralidad, totalmente opuestas a la nuestra, al menos en lo relativo a sus implicaciones primeras. De este modo, la concepción de dignidad o de sacralidad no se comprende necesariamente como afín a una preservación de la existencia, pudiendo incluso devenir en un resultado opuesto. El objeto de respeto o adoración podrá así ser derribado sin temor o reparo alguno por parte del individuo actuante y precisamente con base a la creencia de que toda existencia 
continúa deviniendo en existencia. Hemos escogido este ejemplo como paradigma con el deseo de iluminar más acertadamente los mecanismos profundos de la realidad que deseamos explicar: nos hemos ido a un extremo. Si se quiere rebatir esta idea, naturalmente, se podrá ver aquí también un modelo más a la hora de ejercer un dominio sobre el medio, y sin embargo comprendemos que esta lucha entre el individuo y su entorno se resuelve menos hipócritamente en este caso en tanto que la dialéctica de contrarios no ejerce su dominio sobre la conducta del sujeto, al menos no tan despóticamente, y desde luego la apropiación del medio se realiza con mayor nobleza aun dentro de una concepción trágica de la existencia. Por terminar con esta aclaración, diremos que si en un caso se despoja de dignidad al sujeto para acabar con su existencia, en el otro se le reconoce tal dignidad y luego, llegado el caso aunque dándose prioridad al equilibrio del medio -y este factor resulta determinante en tanto que el sujeto no rechaza su propia condición depredadora pero no abusa de la misma-, se acaba con ella. Como tercera opción quedará el ya aludido maniqueísmo a primera vista aplaudible si bien enteramente sintomático de una neurosis social con sus consiguientes consecuencias: de ello ya se ha hablado por lo que simplemente lo apuntamos.

En función de estas experiencias, cabe indicar que allí donde nuestra cultura cientificista sitúa un poso de respeto y de dignidad, el sujeto se obliga éticamente $-\mathrm{y}$, en su hipertrofia, antinaturalmente- a preservar a toda costa dicha forma de existencia. Llegados a estas latitudes, cabe recordar que en uno u otro momento se puede encontrar existencia en cualquier sustancia hoy comprendida como no existente, como no vital, pues para ello nos bastará con observar inteligencia, lenguaje, sensibilidad, o cualquier capacidad o cualidad desde la que nosotros comprendamos nuestra individualidad, en otro modo de existencia -e incluso en realidades hoy tomadas incluso como inexistentes, pues todo ello forma parte del proceso de terrible atomización que actúa como pulsión dominante en este juego, dándose incluso el caso de que desde esta nivelación por la base sin fundamento ni extremo, se comprenda la mínima sustancia equiparable o consustancial al sujeto, un gen por ejemplo, como poseedora de los derechos y atributos en estas páginas señalados. Esta serie de objetos de derecho, en fin, podrá siempre resultar tan extensa como atributos reconozcamos como dignos o sagrados.

Si de nuevo contrastamos esta realidad con lo observable en una cultura escasamente desarrollada en su faceta técnica como la dibujada líneas atrás -acaso no respetada por nuestros ensimismados ojos a poco que actúen de modo gravoso hacia el medio al menos a nuestro entendimiento-, esa misma 
existencia podrá respetarse y a la vez deponerse en tanto que se comprende lo individual como menos relevante o, si se quiere, secundario respecto del medio. Se participa más equilibradamente, en fin, del juego de la vida y de la muerte. Se conocen las reglas y el sujeto se ajusta a las mismas, sin rebasar sus límites y, por lo tanto, sin necesidad de castigarse y desnaturalizarse por haberlos rebasado. ${ }^{7}$ El concepto de dignidad, en este momento y siguiendo con la idea recién interrumpida, no quedará ya identificado con cuanto de individual hay o reside en cada existencia, siendo así que se podrá llegar al extremo de sacrificar en aras de la 'perpetuación de lo existente' un 'sobreexceso' vital: pero debatir en torno a ello nos llevaría hacia el terreno de la sociología, la antropología y la historia de las religiones, y no es tal el marco de este texto. ${ }^{8}$

Todo ello, como vemos, queda en relación con consideraciones éticas a las que, por unos motivos $\mathrm{u}$ otros, nos vemos fuertemente vinculados y con respecto de las cuales no debemos mostrarnos hipócritas sino a lo sumo rebeldes. De otro modo, resulta obvio, nos debatiríamos en un mar de ira y ceguera en primer lugar en relación con nosotros mismos. El debate hace tiempo que permanece abierto y aquello que observamos constituye sólo la punta de un formidable iceberg, una enorme masa de hielo empujada a la deriva por la voracidad del progreso técnico, una masa a cada momento más y más veloz de modo tal que imposible resulta darle alcance por medio de nuestra actividad reguladora, por medio de nuestras mediciones ético o morales, por mucho que así lo deseemos en tanto que sólo mediante ellas creemos poder liberamos de un concepto altamente elaborado y, en consecuencia, lejano ya de su sentido primero, desprovisto de valor o al menos desprovisto de su naturaleza dual. Las aludidas mediciones o ponderaciones éticas, fragmentando lo escasamente fragmentable en cuanto nos miramos con cierta mesura, esto es, fragmentando al individuo - la condición desde la que nos sentimos

7. Podemos ver que a la hora de retratar estas sociedades no científicas hemos ejemplificado con un modelo ideal, pues conceptos como neurosis, culpa, hybris y un largo etcétera, pertenecen a la realidad humana y se condensan en torno a realidades comunes como el derecho, la religión o tantos otros mecanismos reguladores. Y sin embargo encontramos distinciones cuantitativas en grado tal que llevan a pensar en una plausible diferencia cualitativa. Reside en esta impresión el motivo por el que proponemos los aludidos modelos.

8. Se da, comprendemos, en el sacrificio, una sustitución cualitativa mediante la que el objeto sacrificado redime a una colectividad. Durckheim comprende el sacrificio desde esta misma relación individualidad-generalidad, en tanto que con la supresión de un objeto simbólico se logra una inusual cohesión psíquica. Hubert y Mauss, asimismo, inciden en la función reguladora, social, del sacrificio. 
satisfechos-, se subdividen y ganan cada vez más y más en complejidad, caminando a la carrera, siempre con retraso, en su deseo, nuestro deseo, de dirimir qué es lo correcto, qué lo incorrecto, cuando el objeto que se pretende ponderar hace ya tiempo que permanece fuera de nuestro radio de acción. En última instancia, insatisfechos por lo desacompasado de nuestros esfuerzos racionales a la hora de aprehender una exuberante realidad, podremos al menos reparar en que con nuestro afán no sólo buscamos defender un concepto de justicia sino, acaso primeramente, exculpar nuestras necesidades naturales, incapaces como nos vemos de integrarlas con el medio.

Si engarzamos esto último una vez más con el concepto de culpa, comprenderemos este fenómeno no sólo a modo de autocastigo devenido de una transgresión de la ley natural -mostrando ante nuestro espejo la terrible condición del ser humano en tanto que elemento distanciado de la vida-, sino como elemento diabólico en la medida en que la culpa permite la acción punitiva: existiendo castigo podemos cometer la falta, de manera que aceptando -hipócritamente y como si nos tratásemos de seres movidos por una honda sed de justicia- mayor culpa, nos damos permiso -no necesariamente de modo directo- para multiplicar las acciones punibles. A la hora de realizar estas anotaciones mi pensamiento se dirige hacia la violencia ejercida por el sujeto ante al ecosistema, si bien, parece claro, tal lógica funciona de igual modo en nuestro modo de conducirnos con cualquier otra realidad dado que su naturaleza reside en el ámbito del sujeto.

\section{Posición del sujeto Respecto del medio natural}

A continuación pondremos algunos de los aspectos desarrollados en el último punto en relación con aquellos a priori desde los que partíamos al comienzo de estas páginas, estructuras que, recordemos, determinan nuestro modo de percibir, de estar en y ante la realidad. No parece necesario detenernos en el hecho de que diferentes participaciones espacio-temporales rigen la vida interior de uno u otro organismo. Volviendo a nuestra materia concreta de estudio, por tanto, entendemos que la comprensión que realizamos de la existencia del reino vegetal, dada su diferente participación en relación con los a priori mencionados, siempre será antropocéntrica. Tal desajuste entre la interioridad de un organismo y de otro derivará, en nuestro intento de ponernos en relación con otros modos de vida, en una apreciación manipulada e irreal, radicalmente diferente de la nuestra tanto en sus aspectos cuantitativos 
como cualitativos: todos ellos los comprendemos desde nuestra conciencia existencial, lugar donde ambos fenómenos se distinguen por mucho que queramos buscar y hasta creamos encontrar realidades identificables entre sí. Estas participaciones espacio-temporales gradualmente diferentes de las nuestra, habrán de comprenderse así como una percepción restringida del sujeto que realiza el juicio: sólo tomando como válida dicha apreciación podremos, si lo deseamos, tender puentes entre nosotros y su lejana existencia.

Lo fundamental aquí concierne al hecho de que, a la hora de buscar parámetros con que dotar a las plantas de sensibilidad, lenguaje, motricidad o inteligencia -pudiéndose reducir todo ello a sensibilidad e inteligencia-, no dejamos de hacerlo desde nuestras restringidas concepciones. Por mucho que deseemos, no podemos en modo alguno ponernos en el lugar del objeto de estudio sino sólo en el nuestro, y aunque esto al fin y al cabo puede ser cuanto nos interese pues no buscamos más que un modelo óptimo de relacionarnos con el medio, debemos mostrarnos cautos, aun cuando partamos de los más aprobables propósitos, dados los subterfugios de la razón e incluso de las emociones -precisamente aquellas cualidades por las que creemos sentirnos identificados con el medio vegetal-. Posiblemente reneguemos de ello en tanto que nuestro objetivo no deja de ser, desde una conciencia tranquila, conocer todo, comprender todo, poseer aun intelectivamente todo, perdonar todo. En fin, dirimir todo pero siempre desde nuestro afán y moralidad, y sin embargo la realidad es que el salto entre un orden de existencia y otro resulta inabordable dada la distinta naturaleza de ambos objetos -desde la posesión o no de conciencia y autoconciencia en primer lugar-. Así por tanto, todo cuanto justificamos en relación con las plantas, su merecida dignidad, deviene en tantos casos de un inconsciente interés en justificarnos como individuos.

Por supuesto, de igual modo que las aludidas mediciones escapan a nuestros ojos en cuanto determinan diferencias cualitativas de existencia, lo mismo habremos de señalar en lo concerniente a tantas otras particularidades propias de una condición esencial enteramente ajena a la nuestra, y ello afecta no sólo a nuestra relación con otros organismos sino, como se ha señalado y dando un paso más en esta equiparación, a la mantenida entre distintas culturas, entre individuos de idénticas culturas y, en último término, en nuestra relación con nosotros mismos: el proceso de atomización resulta tan endiabladamente irrefrenable que no hay límite racional que haga frente a su voracidad. Ante ello, más valdría asombrarnos de tal alteridad desde la conciencia -y empatía- de un abismo esencial entre una realidad existencial y otra, que pretender asimilarla enteramente a nuestras capacidades y cualidades, para 
sólo desde ahí, si nos vemos capaces de ello, conferirle un aire de respeto y conformidad con nuestra propia existencia. El aludido proceso, reiteramos, parece imposible de detenerse y en todo caso las soluciones no han de llegar por una vía inasequible sino por la aceptación de nuestra lógica destructiva y, a partir de ahí, buscar una respuesta. Nos adentramos ahora en el ámbito del derecho y de la técnica y por lo tanto salimos con la misma rapidez con la que hemos entrado.

Volviendo al lugar abandonado antes de nuestro último desvío, encontramos que junto a un proceso de relativización y antropocentrismo, se da un endiosamiento del paradigma técnico-científico, aunque ello chocará con nuestros deseos emocionales más reconocibles, más palpables e iluminados por nuestra conciencia, orientados todos ellos a la aparente creación de relaciones sospechosamente humanas ahí donde en tantas ocasiones se siguen introduciendo modelos colonizadores de existencia. ${ }^{9}$ Ante esta actitud quizás

9. Quizás se advierta esto de modo más patente si reparamos en cuánto se da de fenómeno colonizador, de modelo de apropiación de experiencias arraigadas en terreno ajeno, en los fenómenos de fusión cultural tan comunes en nuestro marco occidental (música, cine, etc.), así como en las experiencias religiosas fundamentalmente ligadas a una espiritualidad de origen oriental, dado que ahí donde nos sentimos fuertemente vinculados con realidades, símbolos y pulsiones elementales culturalmente -no esencialmente, claro está- distintas a la propia, se desvela por lo común un proceso de apropiación apenas camuflado y consentido no sólo desde el colonizador sino incluso en ocasiones desde el colonizado -dándose así un proceso de retroalimentación con propiedades homeostáticas-. Cuanto llega hasta nosotros es un hibridismo a menudo forzado a modo de incorporación de formas y estructuras de pensamiento ajenas en nuestro propio imaginario.

Lo que se da aquí, por tanto, consiste en una adaptación de experiencias remotas a nuestro modo de comprensión y percepción, constituyendo la presente actividad una forma de encuentro y posible desafío entre las diferentes culturas desde unos niveles regidos casi exclusivamente por las leyes del mercado. El sentido se pierde, el árbol crece en un terreno inadecuado y, cuando reparamos en ello, dejamos de lado dicha experiencia para colonizar -y paralelamente ser colonizados- otras muchas realidades envolventes. Conocer cuánto de deseo hay en ello y cuánto de necesidad, queda fuera de nuestro alcance. Con todo, resulta conveniente al menos percibir el fondo de la cuestión, pues allí donde creemos abrazar una realidad esencial participamos de lleno de un deseo de dominio y en ocasiones de un falso sometimiento por parte del dominado, un juego en el que la directriz no coincide ya con la satisfacción del sujeto sino con la cantidad de valores tangibles que habrán de recibirse a cambio.

En relación con la dualidad colonizador-colonizado en lo concerniente a los reinos animal y vegetal, señala Francis Hallé: "las plantas y los animales ocupan posiciones bien diferentes en lo que los ecólogos denominan cadenas alimentarias, que se representan tradicionalmente con las plantas abajo y los animales arriba; pero esta ascendencia bastante 
ineludible y respecto de la que podríamos comprendernos más como objetos que como sujetos, se llega sin duda a existencializar la nada así como a subsumir la concepción de ésta en el terreno de la tecnificación, a sacrificarla, quedando para nosotros -escasamente existentes- como toda posesión un mundo desmadejado: dioses sin reino, sin yo e incluso sin otro.

Estas dos últimas opciones, llevadas a un extremo, conducen a dos terrenos sobre los que no podríamos sino echar por tierra nuestro concepto heredado de ética, desterrar esta palabra al basurero de las ensoñaciones e incluso devenir o aceptarnos como seres deshumanizados o, diríamos más, como pura y desnuda naturaleza. No iremos tan lejos.

Como se puede advertir, no caminamos sobre un terreno dado a la estabilidad, sino más bien sobre un cristal completamente pulido y a cada poco más y más inclinado. La pendiente de este último, llegado cierto punto, poco nos podrá importar e inevitablemente habremos de comprender que el movimiento -y la caída no siempre es un obstáculo pues hay que poner todo en relación con una perspectiva a veces no racional, a veces no asimilable- no lo producimos nosotros mismos sino que formamos parte de él.

La asimilación expuesta desde nuestro 'ser diferentes', nos lleva así al reino de las posibilidades: la de, en primer lugar y a medida que los ojos de nuestros instrumentos de medida y conocimiento se agudizan más y más, reparar en la existencia de atributos asimilables a la realidad de lo humano en cualquier átomo del universo, en cualquier organismo vivo o divino, quedando dentro de nuestros puños una enorme nada pues, de acontecer tal situación, todo se habrá reducido a mediciones y aproximaciones a lo real reducidas a su vez a datos confeccionados a nuestra humana escala. Así, si esta participación se fundamenta y acepta desde un plano ético siempre reconfortante en función de los valores morales reiterados una y otra vez a lo largo de este artículo, nos queda por preguntarnos si no poseemos otros medios de participación que no vengan mediados por una visión antropocéntrica a todas luces voraz. En última instancia, cabe suponer, nuestra mirada analítica se arrojará sobre el propio ser para descubrir en sí mismo un compuesto de átomos paradójicamente más y más fragmentados a medida que la lente se perfecciona. ${ }^{10}$ De llegar el momento, la realidad -que seguirá estando ahí-,

triunfal oculta, al mismo tiempo, la autonomía de la base y la dependencia de la cima" (HALLÉ, F., Elogio de la planta. Por una nueva biología, Bilbao, Libros del Jata, 2016, p. 309).

10. Aleccionadora resulta en este sentido la visión de D’Arcy Thompson, quien, ya de paso, diremos, remite tanto a Goethe como a Geoffroy como exponentes de su misma línea de pensamiento: "Cuando analizamos una cosa descomponiéndola en sus partes 
se presentará sin embargo reducida a nuestros ojos al número, la presencia reducida a la ausencia en tanto que, como comentábamos al inicio de estas líneas, el mundo se nos presenta de una forma u otra en función del modo de percepción desde el que nos acerquemos a él.

A la hora de hacer una valoración de lo expuesto y antes de condensar en el siguiente epígrafe los puntos clave de nuestro recorrido, podemos comprender que aquellos factores que nos llevan a empatizar, a dotar-como si con poner una etiqueta todo se arreglase- de un grado de 'ser respetable' al mundo vegetal, pasan por una adecuación de las observaciones tomadas a magnitudes y órdenes asequibles a nuestro discernimiento, aptos todos ellos para nuestra disección intelectual pero en ningún caso adecuados como modo de participación en su realidad dado que diferimos en naturaleza. En este sentido, cabe destacar que desde dicha aproximación no dejamos de trabajar desde la cadena de engranaje de nuestras mediciones lógico-culturales -y que éstas caminan de la mano de nuestros, en tantas ocasiones, ventajistas y desacompasados fundamentos éticos-. Siendo esto así, podemos concluir que un endurecimiento de nuestra visión sólo podrá derivar en una ceguera en contradicción con los principios morales desde los que hemos partido en este texto, si bien es preferible una tentativa de encuentro entre diferentes realidades esenciales aun determinada por la mirada 'dominante', que el no reconocer ningún grado de semejanza entre existencias de distinta naturaleza.

\section{Conclusión}

Cuanto se ha querido exponer en este trabajo, puede condensarse en los siguientes puntos:

- Por una parte, asistimos actualmente a una reformulación de la esencia del mundo vegetal y para ello nos apoyamos en la adjudicación a dicho reino y a partir de nuestra experiencia empírica, de cualidades comprendidas hasta

o en sus propiedades, tendemos a agrandarlas, a exagerar su independencia aparente, y a ocultarnos (al menos por un tiempo) la integridad esencial y la individualidad de la composición en su conjunto. Dividimos el cuerpo en sus órganos, el esqueleto en sus huesos, exactamente de la misma forma en que hacemos un análisis subjetivo de la mente, de acuerdo con las enseñanzas de la psicología, en los factores que la componen: pero sabemos muy bien que juicio y conocimiento, coraje o gentileza, amor o miedo, no tienen una existencia por separado, sino que, de algún modo, son meras manifestaciones o coeficientes imaginarios, de un todo más complejo" (Thompson, D., Sobre el crecimiento y la forma, Madrid, Akal, 2011, pp. 252-253). 
ahora por lo común como propias del reino animal: motricidad, sensibilidad, lenguaje o inteligencia.

Con la presente identificación, reelaboramos a su vez nuestra concepción de las mencionadas aptitudes a la hora de comprendernos a nosotros mismos. De este modo, por ejemplificar, el hallazgo de una variante intelectiva en el reino vegetal viene de la mano de la emergencia en el sujeto de un patrón intelectivo comprendido como irrelevante hasta el momento, o una percepción de distintas modalidades sensitivas en el ámbito del reino animal llega acompañada de la extensión de las cinco facultades hasta ahora entendidas como suficientes para entender nuestra relación entre el cuerpo y nuestro medio. ${ }^{11}$ La adjudicación a otros modos de vida de cualidades en un momento dado exclusivas del ser humano, encuentra su par correspondiente en la comprensión de nuestras facultades desde diferentes ángulos. A su vez, advertir nuevos modos de conocimiento en nosotros mismos nos permite reparar en cualidades complementarias en otros organismos.

- Por otra parte, con objeto de establecer una relación más armónica entre nuestra realidad y, en este caso, el reino vegetal -si bien esto es algo que resulta ampliable a todos los ámbitos existenciales-, nos asimilamos desde nuestra base orgánica -no desde nuestra lógica racional-a otros órdenes de vida. En este sentido, conceptos como respeto o dignidad dejan de identificarse con fenómenos hasta hace poco entronizados relativos al razonamiento, el lenguaje o un concreta sensorialidad, ${ }^{12}$ para asentarse sobre otras distintas capacidades comprendidas por lo común como variaciones de aquéllas. De esta manera, nos fijaremos ante todo en el modo en que el organismo vegetal consigue, mediante sus propios mecanismos, llevar a cabo funciones y actividades que nosotros o bien realizamos de manera diferente o bien incluso no realizamos: entre las primeras encontramos el lenguaje, el movimiento físico a gran velocidad o la lógica; entre las segundas, por ejemplo, la fotosíntesis -si bien la asimilamos a nuestra alimentación y observamos en el modo de

11. Sin detenernos en ello, resulta habitual hoy día, particularmente en un ámbito científico, oír hablar de veintitantos o incluso más de treinta sentidos perceptivos en el ser humano.

12. Al respecto, señala Mancuso: "Para los vegetales, en cambio, la cuestión de la velocidad es de todo punto irrelevante. Si bien el entorno en el que vive una planta también puede enfriarse, calentarse o llenarse de depredadores, la presteza de la respuesta animal no tiene para ella ningún sentido. Le interesa más encontrar una solución eficaz al problema, algo que le permita sobrevivir a pesar del calor, el frío o los depredadores. Para salir bien librado de tan difícil misión, es preferible contar con una organización descentralizada” (Op. Cit., 2017, p. 138). 
llevarla a cabo en situaciones complicadas un rasgo de inteligencia, de búsqueda de soluciones en situaciones de emergencia. $Y$ aun pese a todo, siempre quedará como telón de fondo la noción de conciencia y autoconciencia.

- En paralelo a esto último, términos entre los que encontramos el de individualidad, especie, vida y muerte, son valorados desde nuestro terreno aun reconociéndose una diferencia de naturaleza esencial, ontológica, entre unos organismos y otros, en especial en lo que respecta a aquéllos pertenecientes a diferentes reinos. Que hablemos, por tanto, de muerte o de individualidad desde nuestros patrones nos sirve para comprendernos primeramente a nosotros mismos pero no para adentrarnos en la realidad del reino vegetal. ${ }^{13}$

13. Esta distinción tocante a nuestra comprensión de individualidad, señalada de continuo por Mancuso en sus trabajos, la encontramos desarrollada hoy profusamente en los estudios en torno al reino animal dada la convergencia entre el comportamiento de especies altamente organizadas, el comportamiento de nuestra red neuronal, así como el de internet -comprendido a día de hoy como un segundo cerebro y a corto plazo como el primero-. La cuestión resulta con todo perenne y rescatamos al respecto las palabras de otro dramaturgo, Maeterlinck, cercano al aludido Strindberg y emparentado con la línea organicista en cuyo eje situamos a Goethe y posteriormente a los estudios antroposóficos desarrollados en el XIX y XX. De estos estudios cuanto nos interesa primeramente toca a la relación entre forma y función tan del agrado de Hallé y, yendo hacia atrás en el tiempo y por citar dos ineludibles en la materia, de D’Arcy Thompson y Ernst Haeckel, quien ya al inicio de una de sus obras capitales, Die Welträtsel, asienta su sistema monista sobre una materia espiritualizada y un espíritu animado tanto sensitiva como intelectivamente.

Destacamos al respecto algunas opiniones de Maeterlinck cercanas a nuestra perspectiva y valiosas tanto por su elevada intuición como por su capacidad para ser trasladadas al núcleo del presente trabajo: "el hormiguero debe ser considerado como un individuo único, cuyas células (al contrario de lo que ocurre con las de nuestro cuerpo, que tiene sesenta trillones, aproximadamente) no están aglomeradas, sino disociadas, diseminadas, exteriorizadas, sin dejar de permanecer sometidas, a pesar de su aparente independencia, a la misma ley central. También es posible que se descubra cualquier día una red de relaciones electromagnéticas, etéreas o psíquicas, de las cuales apenas si tenemos una muy vaga noción" (MaeterLinck, M., La vida de las hormigas, Barcelona, Ariel, 2018, pp. 25-26). Poco después, continúa el autor: "Somos, solamente, un ser colectivo, una colonia de células sociales, pero ignoramos quién manda, dirige, reglamenta y armoniza la actividad prodigiosamente compleja y diseminada de nuestra vida orgánica, base de una manifestación accesoria, tardía, precaria y efímera. Si no conocemos ni vemos nuestro propio misterio, que parece saltarnos a la visa, ¿cómo hemos de confiar en descubrir el gran misterio análogo que se oculta en las colonias de los insectos sociales?” (Ibid., p. 27). Por último, como abstracción de lo expuesto, concluye: "Por no ser, como ellas [las hormigas], física e irresistiblemente altruistas, los hombres hemos evolucionado en sentido inverso. Hemos preferido antes que la inmortalidad colectiva la individual. Ahora empezamos a dudar de que ésta sea posible y, entretanto, hemos perdido la noción de aquélla. ¿Volveremos a hallarla?” (Ibid., p. 177). 
En este punto hemos encontrado más franco el acercamiento a otros organismos por parte de culturas menos avanzadas técnicamente y acaso más espiritualmente. En el seno de estas sociedades a las que aún nos referimos a la hora de designarlas como menos avanzadas, conceptos como sacralidad, sacrificio o culpa, desarrollan un papel ajustado a la vida y no tanto a unas leyes éticas comprendidas en tantas ocasiones como ventajistas. En cualquier caso, los fenómenos mencionados cumplen un papel más efectivo, más reparador, que en sociedades como la nuestra donde las realidades rápidamente devienen en conceptos y éstos, con no menos velocidad, se adhieren a maniqueas elaboraciones morales tendentes a paralizar el dinamismo del sujeto.

- Todo ello deriva en la necesidad de revisar aspectos éticos en lo que concierne a nuestra relación con otras especies y organismos e, hipotéticamente, incluso en nuestra relación con realidades comprendidas a día de hoy como no vivientes. Con todo, mientras relativizamos nuestro poder y se conforma un marco ético y a la postre legal de protección de nuestro medio, nuestra naturaleza especulativa, racional, arroja de antemano sus tentáculos hacia nuevas presas en la medida en que el solo hecho de inmiscuirnos, de entrometernos en su condición esencial, acaba por devenir en un previsible fenómeno de apropiación paralelo a dicha intención primera. En este sentido, cabe comprender que todo hallazgo pronto escapa de las manos y la visión del observador neutro, pasando a devenir al poco en un nuevo objeto de apropiación por parte del espíritu técnico. A un mismo tiempo, buscaremos un nuevo elemento de diferenciación entre el ser humano y el medio, desde el que evidenciar una relación de superioridad pues, al fin y al cabo, aquí quien habla o quien cree hacerlo, quien expone sus demandas y su visión del mundo, es el sujeto explorador y no el sujeto explorado. Pudiendo sólo percibir cuanto en primer lugar deseamos percibir, reconociendo cuanto de antemano queremos reconocer, la mirada empírica y científica se revela como un instrumento en modo alguno objetivo pues la presunta objetividad que nos presenta viene determinada, guiada, de antemano, además de situar los hallazgos en la esfera de una realidad tan sólidamente estructurada que su forma es a fin de cuentas la determinada por nuestra comprensión de lo real, nuestra visión del universo. Esto es así hasta el punto de que sólo tomamos como válidas las realidades que vienen a completar nuestro puzle y desechamos sin siquiera reparar en ello lo que no se adecua a la forma estructural que ofrecemos.

- Como corolario podemos mencionar que todo aquello que mediante la técnica se redime, en aras de la técnica se sacrifica, de manera que empatía y 
usurpación caminan a la par pues el mecanismo de fondo requiere o al menos se sirve de ambas dinámicas. Renegar de este mecanismo, consideramos, ya no resulta posible. El problema ha de resolverse, no parece haber otro modo, desde el interior de esta dialéctica y no a sus espaldas, siendo así que luchando contra ella luchamos contra la estructura de nuestra naturaleza y nuestro apéndice lógico-científico. Acaso como último reducto humano nos queda un gesto de aceptación, de estoica sumisión ante una realidad -posthumana- venidera, y la constatación de que la realidad, por mucho que nos volvamos locos por apresarla, descansa completamente fuera o alrededor de uno mismo.

A la hora de aplicar los conceptos que hemos venido empleando y de referirnos al ser humano, al reino vegetal o al animal, hemos optado por generalizar en tanto que, una vez abierta la veda de la escrupulosa diferenciación, las disyuntivas surgen a cada paso y con ello una percepción más falsa de la realidad. En este punto cuanto nos interesa es la visión de conjunto: lo que cohesiona y no lo que diferencia, esto es, no una percepción atomista de alcance potencialmente infinito. Aquello que se ha pretendido es mostrar un conflicto y una de sus derivas.

Ponemos punto y final a estas páginas con la siguiente propuesta de discusión: ¿hasta qué punto variar nuestra percepción de lo real nos da derecho a sublimar unas realidades, unas existencias, que no necesitan de nuestra consideración para existir, sino meramente ser respetadas tal y como son? De esta pregunta se deriva el que tendemos a respetar no simpáticamente sino racionalmente. Otro aspecto tratado nos lleva a abrir la siguiente interrogación: ¿somos conscientes a la hora de defender una concreta realidad de que esa defensa forma parte de un mecanismo respecto del cual su proceder resulta indisociable y cuyos efectos en ocasiones se revelan como opuestos al objetivo en un principio buscado? Si no es posible cortar este hilo pues, o bien pertenece a la misma esencia de la naturaleza o, si lo queremos, forma parte de ese monstruo técnico por el que no sólo nos hemos visto devorados sino del que ya formamos parte, ¿de qué soluciones dispone el individuo para sustraerse a este mecanismo? Aquí surgirán las sombras, pues si parece obvio que los mecanismos reguladores de defensa, legislativos, avanzan siempre con un paso de retraso a costa de no resultar despóticos o reaccionarios -además de tener que ir, querámoslo o no, siempre a remolque de la naturaleza-, más claro comienza a parecernos que la posibilidad de superación de esta entropía saturnal pasa por una reparación o una salida del problema desde dentro, desde nuestra realidad técnica, e incluso, no podemos ser ciegos a este 
devenir, desde nuestra asimilación a ella, desde nuestra domesticación por ella. ¿Tendremos derecho a quejarnos de llegar tal momento? Parece obvio que no es una cuestión de derecho sino de resignación, o acaso de supervivencia. Podremos responder como queramos, pero en el presente devenir el sujeto se revela como objeto de la historia y no como factor libremente actuante. Así, del mismo modo que el Neanderthal y otras pocas especies y subespecies humanas fueron desplazadas por el Sapiens Sapiens -dentro de una cadena que ha de remontarse hasta el comienzo de la vida- permaneciendo en este último una proporción de Neanderthal en su ADN, quizás hemos de comprender un destino similar para nosotros en nuestra relación con futuros herederos. Esto, con suerte. De ser todo ello así, como última reminiscencia humana y de acuerdo con los factores humanos desde los que nos ayudamos a creer dominar el mundo, a creer dominar la naturaleza toda y nuestra propia existencia, al menos nos quedará la culpa.

\section{ReFERENCIAS}

DeSalle, R. / Tattersall, I. (2017). El cerebro. Big Bangs, comportamientos y creencias, Barcelona, Galaxia Gutenberg.

DiÉGuez, A. (2018). Transhumanismo, Barcelona, Herder.

Durkheim, E. (2003). Las formas elementales de la vida religiosa, Madrid, Alianza.

Haeckel, E. (2016). Die Welträtsel: Gemeinverständliche Studien über monistische Philosophie, Berlin, Hofenberg.

Hallé, F. (2016). Elogio de la planta. Por una nueva biología, Bilbao, Libros del Jata.

Julius, F. H. (2008). Metamorfosis. Claves para la comprensión del desarrollo vegetal y de la vida bumana, Buenos Aires, Antropomórfica.

Maeterlinck, M. (2018). La vida de las hormigas, Barcelona, Ariel.

Mancuso, S. (2015). Sensibilidad e inteligencia en el mundo vegetal, Barcelona, Galaxia Gutenberg.

Mancuso, S. (2017). El futuro es vegetal, Barcelona, Galaxia Gutenberg.

Mauss, M. y Hubert, H. (2010). El sacrificio. Magia, mito y razón, Buenos Aires, Las cuarenta.

Strindberg, A. (2016). Una mirada al Universo, Madrid, Siruela.

Thompson. D. (2011). Sobre el crecimiento y la forma, Madrid, Akal. 
